\title{
A Class of Cubic Splines Obtained Through Minimum Conditions
}

\author{
By D. Bini and M. Capovani
}

\begin{abstract}
A class of cubic spline minimizing some special functional is investigated. This class is determined by the solution of a quadratic programming problem in which the minimizing function depends linearly on a parameter $\alpha<2$. For $\alpha=1 / 2$ natural splines are obtained. For $\alpha=-1$ the spline minimizing the mean value of the third derivative is obtained. It is shown that this spline has the best convergence order.
\end{abstract}

1. Introduction. Suppose that we are given a function $f \in C^{4}[a, b]$ and a mesh $\Delta_{n}=\left\{a=x_{0}<x_{1}<\cdots<x_{n}=b\right\}$, such that $x_{i}=x_{0}+i h, h=(b-a) / n$. A function $s_{n}(x)$ is called a cubic spline interpolant to $f(x)$ with respect to $\Delta_{n}$ if:

$$
\begin{aligned}
& s_{n} \text { is a cubic polynomial on }\left[x_{i-1}, x_{i}\right], \quad i=1,2, \ldots, n ; \\
& s_{n} \in C^{2}[a, b] ; \\
& s_{n}\left(x_{i}\right)=y_{i}, \quad y_{i}=f\left(x_{i}\right), \quad i=0,1, \ldots, n .
\end{aligned}
$$

The importance of cubic spline interpolation is described in detail in [1]. Conditions (1.1) lead to a system of $n-1$ equations and $n+1$ unknowns. Two more equations can be given in various ways. For example, if we know $f^{\prime}\left(x_{0}\right)$ and $f^{\prime}\left(x_{n}\right)$ or $f^{\prime \prime}\left(x_{0}\right)$ and $f^{\prime \prime}\left(x_{n}\right)$ we can set $s_{n}^{\prime}\left(x_{i}\right)=f^{\prime}\left(x_{i}\right)$ or $s_{n}^{\prime \prime}\left(x_{i}\right)=f^{\prime \prime}\left(x_{i}\right), i=0, n$, obtaining in this way convergence of $s_{n}^{(i)}(x)$ to $f^{(i)}(x)$ of order $O\left(h^{4-i}\right), i=0,1,2,3$ uniformly over $[a, b][5]$. In the absence of such information, usually the following equations are considered

$$
s_{n}^{\prime \prime}\left(x_{0}\right)=s_{n}^{\prime \prime}\left(x_{n}\right)=0 .
$$

The kind of spline function obtained in this way is known as natural spline, and it is the spline interpolant to $f(x)$ minimizing the functional

$$
F_{2}(s)=\int_{a}^{b}\left(s^{\prime \prime}(x)\right)^{2} d x
$$

which is related to the mean curvature of the graph of the function $s(x)$. For natural spline functions the following result holds [4]

$$
\begin{array}{ll}
\left|s_{n}^{(i)}(x)-f^{(i)}(x)\right|=O\left(h^{2-i}\right), & a \leqslant x \leqslant b, i=0,1 \\
\left|s_{n}^{(i)}(x)-f^{(i)}(x)\right|=O\left(h^{4-i}\right), & \tilde{a} \leqslant x \leqslant \tilde{b}, i=0,1,2 \\
& \tilde{a}-a=b-\tilde{b}=O(h \log h) .
\end{array}
$$

Received December 13, 1983; revised February 20, 1984 and February 4, 1985.

1980 Mathematics Subject Classification. Primary 41Ai5, 65D05, 65D07: Secondary 90C20.

1986 American Mathematical Society $0025-5718 / 86 \$ 1.00+\$ .25$ per page 
Recently, another variational condition has been analyzed [3]. The spline minimizing the functional

$$
F_{1}(s)=\int_{a}^{b}\left(s^{\prime}(x)\right)^{2} d x
$$

has been shown useful for computational purposes.

In this paper we consider cubic splines which minimize some special functionals. This set of splines is obtained by considering the functionals $F_{1}$ and $F_{2}$, two other functionals $F_{0}$ and $F_{3}$, involving the functions $s(x)$ and $s^{\prime \prime \prime}(x)$ respectively, and convex combinations of $F_{0}, F_{1}, F_{2}$ and $F_{3}$.

In Section 2, we show that such splines can be obtained by solving a quadratic programming problem in which the minimizing function depends linearly on a parameter $\alpha,-1 \leqslant \alpha \leqslant 31 / 32$. In particular, for $\alpha=1 / 2$ we find the natural spline, for $\alpha=7 / 8$ the function minimizing $F_{1}$. We widen the class by showing that for any $\alpha \leqslant 1$ the quadratic programming problem has a solution and that there exists a positive integer $n_{0}$ such that the quadratic programming problem has a solution for any $n \geqslant n_{0}$ if and only if $\alpha<2$.

In Section 3, using the properties of a special class of matrices defined in [2], we determine the best spline interpolating $f$ in the class of splines depending on $\alpha$, $\alpha<2$. We show that the best spline in this class is the spline which minimizes the functional

$$
F_{3}(s)=\sum_{i=0}^{n-1} \int_{x_{i}}^{x_{i+1}}\left(s^{\prime \prime \prime}(x)\right)^{2} d x,
$$

obtained with $\alpha=1$. For this spline we get the following result

$$
\begin{array}{ll}
\left|s_{n}^{(i)}(x)-f^{(i)}(x)\right|=O\left(h^{3-i}\right), & a \leqslant x \leqslant b, i=0,1,2 ; \\
\left|s_{n}^{(i)}(x)-f^{(i)}(x)\right|=O\left(h^{4-i}\right), & \tilde{a} \leqslant x \leqslant \tilde{b}, i=0,1,2,3, \\
& \tilde{a}-a=b-\tilde{b}=O(h \log h) .
\end{array}
$$

Thus, one gains one order of convergence and the convergence of one more derivative, compared to natural splines.

2. Preliminaries. Following Stoer \& Bulirsch [5] we set

$$
\begin{array}{r}
s_{n}(x)=M_{j}\left(x_{j+1}-x\right)^{3} /(6 h)+M_{j+1}\left(x-x_{j}\right)^{3} /(6 h)+\alpha_{j}\left(x-x_{j}\right)+\beta_{j}, \\
x_{j} \leqslant x \leqslant x_{j+1}, j=0,1, \ldots, n-1 ;
\end{array}
$$

so that, from (1.1), we obtain

$$
\begin{aligned}
& \beta_{j}=f\left(x_{j}\right)-M_{j} h^{2} / 6 ; \\
& \alpha_{j}=\left(f\left(x_{j+1}\right)-f\left(x_{j}\right)\right) / h-h\left(M_{j+1}-M_{j}\right) / 6 ;
\end{aligned}
$$

where the $(n+1)$-vector $M=\left(M_{j}\right)$ fulfills the relation

$$
A M=b,
$$


where $A$ is the $(n-1) \times(n+1)$ matrix

$$
A=\left[\begin{array}{ccccccccc}
1 & 4 & 1 & & & & & \\
& 1 & 4 & 1 & & & & \\
& & \cdot & \cdot & \cdot & & & \\
& & & & \cdot & \cdot & & \\
& & & & & 1 & 4 & 1
\end{array}\right],
$$

$$
b=\left(b_{i}\right), \quad b_{i}=6\left(f\left(x_{i+1}\right)-2 f\left(x_{i}\right)+f\left(x_{i-1}\right)\right) / h^{2}, \quad i=1,2, \ldots, n-1 .
$$

Consider the following functionals

$$
\begin{aligned}
& F_{0}(s)=\sum_{i=0}^{n-1} \int_{x_{i}}^{x_{i+1}}\left(s(x)-r_{i}(x)\right)^{2} d x \\
& r_{i}(x)=\left(x-x_{i}\right)\left(y_{i+1}-y_{i}\right) /\left(x_{i+1}-x_{i}\right)+y_{i} \\
& F_{1}(s)=\int_{a}^{b}\left(s^{\prime}(x)\right)^{2} d x \\
& F_{2}(s)=\int_{a}^{b}\left(s^{\prime \prime}(x)\right)^{2} d x \\
& F_{3}(s)=\sum_{i=0}^{n-1} \int_{x_{i}}^{x_{i+1}}\left(s^{\prime \prime \prime}(x)\right)^{2} d x .
\end{aligned}
$$

The functional $F_{0}^{1 / 2}$ is related to the area included between the graph of $s(x)$ and the line obtained by connecting the point $\left(x_{i}, y_{i}\right)$ to the point $\left(x_{i+1}, y_{i+1}\right), i=$ $0,1, \ldots, n-1$. The spline which minimizes $F_{0}$ is as close as possible to this polygonal line.

The functional $F_{1}^{1 / 2}$ is an average of the slope of the graph of $s(x)$ and the spline which minimizes $F_{1}$ has been analyzed in [3].

The functional $F_{2}^{1 / 2}$ is related to the mean curvature of the graph of $s(x)$ and the spline minimizing $F_{2}$ is known as the natural spline; it satisfies $s^{\prime \prime}\left(x_{0}\right)=s^{\prime \prime}\left(x_{n}\right)=0$.

The functional $F_{3}^{1 / 2}$ has a less easy geometric interpretation and we can look at it as a number related to the mean curvature of the graph of $s^{\prime}(x)$.

Other functionals can be obtained by taking any convex combination of $F_{i}(s)$, $i=0,1,2,3$.

By direct computation we can show the following result:

PROPOSITION 1. Consider the quadratic programming problem:

$$
\left\{\begin{array}{l}
\operatorname{Min} M^{T} A_{\alpha} M \\
A M=b
\end{array}\right.
$$

where $A$ and $b$ are defined in (2.4), $M=\left(M_{i}\right), i=0,1, \ldots, n$, is an $(n+1)$-vector and $A_{\alpha}$ is the $(n+1) \times(n+1)$ matrix given by

$$
A_{\alpha}=\left[\begin{array}{ccccccccc}
1 & \alpha & & & & & & \\
\alpha & 2 & \alpha & & & & & \\
& \alpha & 2 & & & & & \\
& & . & . & . & . & & \\
& & & . & . & . & \\
& & & & & & 2 & \alpha \\
& & & & & & \alpha & 1
\end{array}\right] .
$$


Then (2.6) has a solution if $-1 \leqslant \alpha \leqslant 31 / 32$ and this solution defines, through (2.1), (2.2), a cubic spline interpolating $f(x)$ which minimizes the functional

$$
\begin{aligned}
& F_{0} \quad \text { if } \alpha=31 / 32, \quad F_{1} \quad \text { if } \alpha=7 / 8, \\
& F_{2} \quad \text { if } \alpha=1 / 2, \quad F_{3} \quad \text { if } \alpha=-1, \\
& \sum_{i=0}^{3} \theta_{i} F_{i}, \quad \text { if }-1 \leqslant \alpha \leqslant 31 / 32, \text { for some } \theta_{i}=\theta_{i}(\alpha) \geqslant 0 .
\end{aligned}
$$

A similar result holds also in the case in which the knots $x_{i}$ are not uniformly spaced.

It is interesting to point out that the same matrix formulation (2.6) allows us to deal with all the different conditions given in (2.5).

Two questions arise at this point:

- Are there other values of $\alpha$, besides those in the range [-1,31/32], for which the problem (2.6) has a solution?

- Is there a best value of $\alpha$ in the range of the feasible values, for which the convergence of $s_{n}^{(i)}$ to $f^{(i)}$ is best possible?

In order to deal with these two questions we introduce a special class of matrices which has been used in [2].

Let $\tau_{m}$ be the linear space spanned by the set $\left\{I, H, H^{2}, \ldots, H^{m-1}\right\}$, where $H=\left(h_{i, j}\right)$ is the $m \times m$ matrix such that $h_{i, i+1}=h_{i+1, i}=1, i=1,2, \ldots, m-1$, $h_{i, j}=0$ otherwise. Observe that $\tau_{m}$ is closed under the row-column product, and $A B=B A$ for any $A, B \in \tau_{m}$, that is, $\tau_{m}$ is a commutative algebra; moreover, $A \in \tau_{m}$ if and only if

$$
A H-H A=0 .
$$

Writing down condition (2.7), we get

$$
\begin{aligned}
& a_{i, j+1}+a_{i, j-1}=a_{i+1, j}+a_{i-1, j}, \\
& a_{0, j}=a_{i, 0}=a_{m+1, j}=a_{i, m+1}=0,
\end{aligned} \quad i, j=1,2, \ldots, m .
$$

The relation, called cross-sum condition, allows us to build up all the entries of any matrix $A \in \tau_{m}$ starting with the first row, or the first column, of $A$. Moreover, we have the following properties [2]:

If $A \in \tau_{m}$, then:

$A$ is symmetric, i.e., $a_{i, j}=a_{j, i}$;

$A$ is persymmetric, i.e., $a_{i, j}=a_{m-i+1, m-j+1}$;

If $F=(\sqrt{2 /(m+1)} \sin (\pi i j /(m+1)))$ then $F$ is

symmetric and orthogonal and $F A F$ is diagonal;

The eigenvalues of $H$ are given by $2 \cos (\pi i /(m+1))$.

Now consider problem (2.6) and partition the matrices $A$ and $A_{\alpha}$ in the following way:

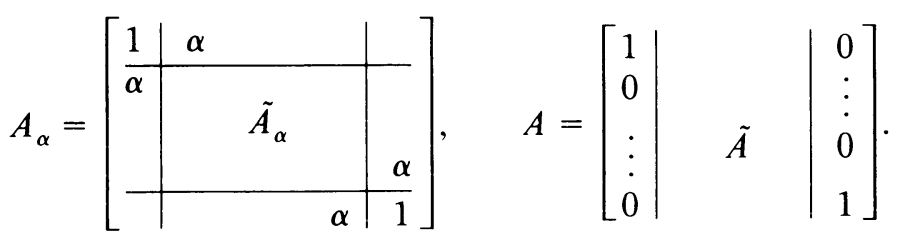


Since

$$
\tilde{A}_{\alpha}=2 I+\alpha H, \quad \tilde{A}=4 I+H,
$$

we have $\tilde{A}_{\alpha}, \tilde{A} \in \tau_{m}, m=n-1$. Expressing the components $M_{1}, M_{2}, \ldots, M_{n-1}$ as a function of $M_{0}$ and $M_{n}$, by using the constraints $A M=b$ and substituting in the quadratic function, we get

$$
\begin{gathered}
M^{T} A_{\alpha} M=\left(M_{0}, M_{n}\right)\left[\begin{array}{ll}
\varphi & \psi \\
\psi & \varphi
\end{array}\right]\left[\begin{array}{c}
M_{0} \\
M_{n}
\end{array}\right]-2\left(M_{0}, M_{n}\right)\left[\begin{array}{c}
p_{1} \\
p_{2}
\end{array}\right] ; \\
\varphi=e_{1}^{T} B_{\alpha} e_{1}=e_{n-1} B_{\alpha} e_{n-1} ; \quad \psi=e_{1}^{T} B_{\alpha} e_{n-1}=e_{n-1}^{T} B_{\alpha} e_{1} ; \\
p_{1}=e_{1}^{T}\left(B_{\alpha}+\alpha \tilde{A}^{-1}-I\right) b ; \quad p_{2}=e_{n-1}^{T}\left(B_{\alpha}+\alpha \tilde{A}^{-1}-I\right) b ; \\
B_{\alpha}=I-2 \alpha \tilde{A}^{-1}+\tilde{A}^{-1} \tilde{A}_{\alpha} \tilde{A}^{-1} \in \tau_{n-1} ;
\end{gathered}
$$

where $e_{1}, e_{n-1}$ are the first and the last columns of the $(n-1) \times(n-1)$ identity matrix. Relations (2.10) hold in view of the symmetry and persymmetry of the matrix $B_{\alpha} \in \tau_{n-1}$.

Since the $2 \times 2$ matrix in $(2.10 a)$ is a principal submatrix of $B_{\alpha}$, it is positive definite whenever $B_{\alpha}$ is positive definite. This fact allows us to prove the following

Proposition 2. For any $\alpha \leqslant 1$, the problem (2.6) has a solution.

Proof. It is sufficient to prove that if $\alpha \leqslant 1$ the matrix $B_{\alpha}$ is positive definite. Now the eigenvalues $\lambda_{i}$ of $B_{\alpha}$ can be obtained by (2.10b), (2.9) and (2.8). We have in fact

$$
\lambda_{i}=1+\left(2-2 \alpha\left(4+c_{i}\right)\right) /\left(4+2 c_{i}\right)^{2}, \quad c_{i}=\cos (\pi i / n) .
$$

Therefore, $B_{\alpha}$ is positive definite if and only if $\alpha<\left(9+2 c_{i}^{2}+8 c_{i}\right) /\left(4+c_{i}\right)$. Now, since $\left(9+2 c_{i}^{2}+8 c_{i}\right) /\left(4+c_{i}\right)>1, i=1,2, \ldots, n-1$, we get the result.

We can further widen the set of $\alpha$ for which problem (2.6) has a solution if we look at this issue from an asymptotic point of view. First we must compute the values to which $\varphi$ and $\psi$ converge as $n$ tends to infinity.

LEMMA 1. We have

$$
\begin{aligned}
& \varphi=(2-\alpha) / \sqrt{3}+\theta_{n}, \quad\left|\theta_{n}\right|<\left(2 /\left(3 n^{2}\right)\right)(1+|\alpha|) \\
& \psi=0+\theta_{n}^{\prime}, \quad\left|\theta_{n}^{\prime}\right|<\left(16 /\left(3 n^{2}\right)\right)(1+|\alpha|) .
\end{aligned}
$$

Proof. Since $B_{\alpha} \in \tau_{n-1}$, from (2.8), (2.9), (2.10b) we have

$$
\begin{aligned}
& F B_{\alpha} F=\operatorname{Diag}\left(\lambda_{1}, \lambda_{2}, \ldots, \lambda_{n-1}\right), \\
& \lambda_{i}=1+\left(2-2 \alpha\left(4+c_{i}\right)\right) /\left(4+2 c_{i}\right)^{2}, \quad c_{i}=\cos (\pi i / n) .
\end{aligned}
$$

Therefore, $\varphi=\varphi_{1}+\alpha \varphi_{2}, \psi=\psi_{1}+\alpha \psi_{2}$, where

$$
\begin{aligned}
& \varphi_{1}=(2 / n) \sum_{i=1}^{n-1}\left(1+2 /\left(4+2 c_{i}\right)^{2}\right) s_{i}^{2}, \\
& \varphi_{2}=(2 / n) \sum_{i=1}^{n-1}\left(\left(8+2 c_{i}\right) /\left(4+2 c_{i}\right)^{2}\right) s_{i}^{2}, \quad s_{i}=\sin (\pi i / n) ; \\
& \psi_{1}=(2 / n) \sum_{i=1}^{n-1}\left(1+2 /\left(4+2 c_{i}\right)^{2}\right)(-1)^{i+1} s_{i}^{2}, \\
& \psi_{2}=(2 / n) \sum_{i=1}^{n-1}\left(\left(8+2 c_{i}\right) /\left(4+2 c_{i}\right)^{2}\right)(-1)^{i+1} s_{i}^{2} .
\end{aligned}
$$


Moreover, the quantities (2.11a) can be viewed as the result of applying the trapezoidal quadrature formula to suitable continuous functions with step $\pi / n$ on the interval $[0, \pi]$. Therefore, we get (see $[5$, p. 121])

$$
\begin{aligned}
& \varphi_{1}=(2 / \pi) \int_{0}^{\pi}\left(1+2 /(4+2 \cos x)^{2}\right) \sin ^{2} x d x+k_{1} /\left(6 n^{2}\right) \\
& \varphi_{2}=(2 / \pi) \int_{0}^{\pi}\left((8+2 \cos x) /(4+2 \cos x)^{2}\right) \sin ^{2} x d x+k_{2} /\left(6 n^{2}\right)
\end{aligned}
$$

where $\left|k_{i}\right|, i=1,2$, can be bounded by the maximum absolute value which the second derivative of the corresponding integrand function takes on over $[0, \pi]$. Therefore, by evaluating the primitives and the second derivatives of these functions, we get

$$
\begin{array}{ll}
\varphi_{1}=2 / \sqrt{3}+k_{1} /\left(6 n^{2}\right), & \left|k_{1}\right| \leqslant 4, \\
\varphi_{2}=-1 / \sqrt{3}+k_{2} /\left(6 n^{2}\right), & \left|k_{2}\right| \leqslant 3 .
\end{array}
$$

Concerning $\psi_{1}$ and $\psi_{2}$, observe that each formula in (2.11b) can be viewed as the difference of two quadrature formulas applied to the same function with the same step, but with different knots. Therefore, we have

$$
\begin{array}{ll}
\psi_{1}=0+4 k_{3} /\left(3 n^{2}\right), & \left|k_{3}\right| \leqslant 4, \\
\psi_{2}=0+4 k_{4} /\left(3 n^{2}\right), & \left|k_{4}\right| \leqslant 3 .
\end{array}
$$

Now we are ready to prove the following

Proposition 3. There exists a positive integer $n_{0}$ such that, for every $n \geqslant n_{0}$, the problem (2.6) has a solution for any $f \in C^{4}[a, b]$ if and only if $\alpha<2$.

Proof. The assertion follows from Lemma 1, since the existence of a solution, for any $f \in C^{4}[a, b]$, is equivalent to the positive definiteness of the matrix $\left[\begin{array}{ll}\varphi & \psi \\ \psi & \psi\end{array}\right]$.

3. Convergence. From relation (2.10a), under the hypothesis $\alpha<2$, we have that the point in which the function $M^{T} A_{\alpha} M$ takes on its minimum value is given by:

$$
\left[\begin{array}{l}
M_{0} \\
M_{n}
\end{array}\right]=\left[\begin{array}{ll}
\varphi & \psi \\
\psi & \varphi
\end{array}\right]^{-1}\left[\begin{array}{l}
p_{1} \\
p_{2}
\end{array}\right]
$$

that is,

$$
M_{0}=-\left(\psi p_{2}-\varphi p_{1}\right) /\left(\varphi^{2}-\psi^{2}\right), \quad M_{n}=-\left(\psi p_{1}-\varphi p_{2}\right) /\left(\varphi^{2}-\psi^{2}\right)
$$

Our aim is to find out how $M_{0}$ and $M_{n}$ depend on $\alpha$, then determine the value of $\alpha$ for which one obtains best convergence. Since we already know $\varphi$ and $\psi$, we have to compute $p_{1}$ and $p_{2}$. As a first step of this evaluation, observe that from $(2.10 \mathrm{~b})$ we have

$$
p_{1}=\sum_{i=1}^{n-1} u_{i} b_{i}, \quad u=\left(u_{i}\right), \quad u=\left(B_{\alpha}+\alpha \tilde{A}^{-1}-I\right) e_{1} .
$$

Now, since $B_{\alpha}+\alpha \tilde{A}^{-1}-I=2(1-2 \alpha) \tilde{A}^{-2}$ we have

$$
u=2(1-2 \alpha) \tilde{A}^{-2} e_{1}
$$


Moreover, since $\tilde{A}^{-2} \in \tau_{n-1}$ is symmetric and persymmetric, we have that the $i$ th component of $\tilde{A}^{-2} e_{n-1}$ is equal to the $i$ th component of $\tilde{A}^{-2} e_{1}$; therefore

$$
\begin{aligned}
p_{1}=2(1-2 \alpha) \sum_{i=1}^{n-1} v_{i} b_{i}, \quad p_{2}=2(1-2 \alpha) \sum_{i=1}^{n-1} v_{n-i} b_{i}, \\
v=\left(v_{i}\right), v=\tilde{A}^{-2} e_{1} .
\end{aligned}
$$

Now our task has been reduced to the computation of $b$ and $v$. As far as the vector $b$ is concerned we have the following

LEMMA 2. If $f \in C^{4}[a, b]$ and $k=\max _{a \leqslant x \leqslant b}\left|f^{(4)}(x)\right|$, then

$$
\begin{array}{r}
b_{i}=6\left(f^{\prime \prime}(a)+i h f^{\prime \prime \prime}(a)+h^{2} \gamma_{i}\right), \quad b_{n-i}=6\left(f^{\prime \prime}(b)-i h f^{\prime \prime \prime}(b)+h^{2} \delta_{i}\right), \\
\left|\gamma_{i}\right|,\left|\delta_{i}\right|<\left(1 / 12+i^{2} / 2\right) k .
\end{array}
$$

Proof. In view of (2.4), applying Taylor's formula to $f(x)$ at the point $x_{i}$ with step $h$ and $-h$ yields

$$
b_{i}=6\left(f^{\prime \prime}\left(x_{i}\right)+\xi_{i} h^{2}\right), \quad\left|\xi_{i}\right|<k / 12 .
$$

Applying Taylor's formula again to the function $f^{\prime \prime}(x)$ at the point $a$ and $b$ with step $i h$ and $-i h$, respectively, yields

$$
\begin{aligned}
& f^{\prime \prime}\left(x_{i}\right)=f^{\prime \prime}(a)+i h f^{\prime \prime \prime}(a)+\left((i h)^{2} / 2\right) \eta_{i}, \\
& f^{\prime \prime}\left(x_{n-i}\right)=f^{\prime \prime}(b)-i h f^{\prime \prime \prime}(b)+\left((i h)^{2} / 2\right) \nu_{i}, \quad\left|\eta_{i}\right|,\left|\nu_{i}\right| \leqslant k,
\end{aligned}
$$

which completes the proof.

Concerning the computation of the vector $v$, we use the properties of class $\tau_{m}$ to prove the following

Lemma 3. Let $d=(2-\sqrt{3})$. Then the vector $v=\tilde{A}^{-2} e_{1}$ satisfies the relation

$$
v_{i}=(-1)^{i+1} i d^{i+1}\left(1-d^{2 n-2 i}\right) /\left(1-d^{2}\right)+\mu_{i}, \quad\left|\mu_{i}\right|<n^{2} d^{n+1} .
$$

Proof. First observe that $\tilde{A}=4 I+H$ and that the matrix $C=4 I-H$ satisfies $C=D \tilde{A} D$, where $D=\operatorname{Diag}\left(1,-1,1,-1, \ldots,(-1)^{n}\right)$. Therefore,

$$
C^{-2}=(1 / 16)\left(\sum_{j=0}^{\infty} H^{j} / 4^{j}\right)^{2}
$$

has positive entries and, since $\tilde{A}^{-2}=D C^{-2} D$, we have $v_{i}=(-1)^{i+1}\left|v_{i}\right|$ where $\left|v_{i}\right|$ are the elements of the first row of $C^{-2}$.

Now, in order to compute $\left|v_{i}\right|$, observe that the elements $c_{i}$ of the first row of $C^{-1}$ are given by $c_{i}=d_{n-i-1} / d_{n-1}$, where $d_{i}$ satisfies the difference equation

$$
d_{i+1}=4 d_{i}-d_{i-2}, \quad d_{0}=1, \quad d_{1}=4 .
$$

By solving the above recurrence, we get $d_{i}=\left(d^{-i-1}-d^{i+1}\right) /(2 \sqrt{3})$, where $d=(2-\sqrt{3})$. Hence

$$
c_{i}=\left(d^{i}-d^{2 n-i}\right) /\left(1-d^{2 n}\right)=d^{i}+\gamma_{i}, \quad\left|\gamma_{i}\right|<d^{n+1} .
$$

Now set $C^{-1}=G+E$, where $G \in \tau_{n-1}$ is defined by its first row $g_{1, j}=d^{j}$. Since $C \in \tau_{n-1}$, then $E \in \tau_{n-1}$, hence $G E=E G$ and, setting $|v|=\left(\left|v_{i}\right|\right)$, we have $|v|=$ $(G+E)^{2} e_{1}=\left(G^{2} e_{1}+w\right), w=2 E G e_{1}+E^{2} e_{1}$. Therefore $\|w\| \leqslant 2\|E\|\left\|G e_{1}\right\|+\|E\| \cdot$ $\left\|E e_{1}\right\|$, where $\|w\|=\max \left|w_{i}\right|$ and $\|E\|=\max _{i} \sum_{j=1}^{n-1}\left|e_{i, j}\right|$. Now, from (3.3) we have $\left\|E e_{1}\right\|<d^{n+1},\left\|G e_{1}\right\| \leqslant d$ and, using the cross-sum condition, $\|E\| \leqslant d^{n+1} n^{2} / 4$, whence $\|w\| \leqslant d^{n+2} n^{2} / 2+d^{2 n+2} n^{2} / 4<n^{2} d^{n+1}$. 
Now, to complete the proof, we must still show that the elements of $z=G^{2} e_{1}$ are given by $z_{i}=i d^{i+1}\left(1-d^{2 n-2 i}\right) /\left(1-d^{2}\right)$.

We proceed by induction on $i$. The result holds for $i=1$ and $i=2$, by construction of $G$. Suppose that it holds for $r$ and $r-1$; by the cross-sum condition we have

$$
\begin{aligned}
z_{r+1} & =\sum_{i=1}^{n-1} d^{i} g_{r+1, i}=\sum_{i=1}^{n-1} d^{i}\left(g_{r, i-1}+g_{r, i+1}-g_{r-1, i}\right) \\
& =d\left(z_{r}-d^{n-1} g_{r, n-1}\right)+d^{-1}\left(z_{r}-d g_{r, 1}\right)-z_{r-1} .
\end{aligned}
$$

Since $g_{r, n-1}=d^{n-r}$, we get

$$
z_{r+1}=z_{r}\left(d+d^{-1}\right)-z_{r-1}-d^{2 n-r}-d^{r}
$$

and, by the inductive assumptions

$$
\begin{aligned}
z_{r+1}= & r d^{r+1}\left(1-d^{2 n-2 r}\right)\left(d+d^{-1}\right) /\left(1-d^{2}\right) \\
& -(r-1) d^{r}\left(1-d^{2 n-2 r+2}\right) /\left(1-d^{2}\right)-d^{2 n-r}-d^{r} \\
= & (r+1) d^{r+2}\left(1-d^{2 n-2 r-2}\right) /\left(1-d^{2}\right),
\end{aligned}
$$

which completes the proof.

From the above lemma we get the following relation:

$$
v_{i}=\left((-1)^{i+1} i d^{i+1} /\left(1-d^{2}\right)+\theta_{i}\right), \quad\left|\theta_{i}\right|<2 n^{2} d^{n+1} .
$$

Now we are ready to compute $p_{1}$ and $p_{2}$. From the above relation, Lemma 2 and (3.2) we have

$$
\begin{aligned}
& p_{1}=12(1-2 \alpha)\left(\sigma f^{\prime \prime}(a)+h \rho f^{\prime \prime \prime}(a)+\omega_{1} h^{2}\right)+O\left(n^{3} d^{n}\right) \\
& p_{2}=12(1-2 \alpha)\left(\sigma f^{\prime \prime}(b)+h \rho f^{\prime \prime \prime}(b)+\omega_{2} h^{2}\right)+O\left(n^{3} d^{n}\right)
\end{aligned}
$$

where

$$
\begin{aligned}
& \sigma=\sum_{i=1}^{n-1}(-1)^{i+1} i d^{i+1} /\left(1-d^{2}\right)+\sum_{i=1}^{n-1} \theta_{i}, \\
& \rho=\sum_{i=1}^{n-1}(-1)^{i+1} i^{2} d^{i+1} /\left(1-d^{2}\right)+\sum_{i=1}^{n-1} i \theta_{i}, \\
& \left|\omega_{1}\right|,\left|\omega_{2}\right| \leqslant k \sum_{i=1}^{n-1}\left(1 / 12+i^{2} / 2\right) i d^{i+1} /\left(1-d^{2}\right) .
\end{aligned}
$$

Now, by using the difference equation technique (see [5, p. 438]) it is easy to prove that

$$
\begin{gathered}
\sum_{i=1}^{n-1}(-1)^{i+1} i d^{i+1} /\left(1-d^{2}\right) \\
=\sqrt{3} / 36+d^{n+1}(n-d /(d+1)) /\left((1+d)\left(1-d^{2}\right)\right), \\
\sum_{i=1}^{n-1}(-1)^{i+1} i^{2} d^{i+1} /\left(1-d^{2}\right) \\
=1 / 36+\left(n^{2} /(1+d)-2 n d /(1-d)^{2}+d(d-1) /(1+d)^{3}\right) d^{n+1} /\left(1-d^{2}\right), \\
\left|\omega_{1}\right|,\left|\omega_{2}\right| \leqslant k / 12 \sum_{i=1}^{\infty} i d^{i+1} /\left(1-d^{2}\right)+k / 2 \sum_{i=1}^{\infty} i^{3} d^{i+1} /\left(1-d^{2}\right) \\
=(k / \sqrt{12})(1 / 24+1)=25 k /(24 \sqrt{12})<k / 3
\end{gathered}
$$


where, for the sake of simplicity, we have assumed $n$ even. Therefore, we have

$$
\begin{aligned}
& p_{1}=(\sqrt{3} / 3)(1-2 \alpha)\left(f^{\prime \prime}(a)+(1 / \sqrt{3}) h f^{\prime \prime \prime}(a)+O\left(h^{2}\right)\right), \\
& p_{2}=(\sqrt{3} / 3)(1-2 \alpha)\left(f^{\prime \prime}(b)+(1 / \sqrt{3}) h f^{\prime \prime \prime}(b)+O\left(h^{2}\right)\right) .
\end{aligned}
$$

From relations (3.4), (3.1) and Lemma 1, we finally get the following

Proposition 4. The second derivatives $M_{0}, M_{n}$ at the points $a$ and $b$, respectively, of the spline function obtained by (2.6), are given by

$$
\begin{aligned}
& M_{0}=((2 \alpha-1) /(\alpha-2))\left(f^{\prime \prime}(a)+(1 / \sqrt{3}) h f^{\prime \prime \prime}(a)\right)+O\left(h^{2}\right), \\
& M_{n}=((2 \alpha-1) /(\alpha-2))\left(f^{\prime \prime}(b)+(1 / \sqrt{3}) h f^{\prime \prime \prime}(b)\right)+O\left(h^{2}\right) .
\end{aligned}
$$

We are now ready to determine the value of $\alpha$ for which one obtains best convergence of $s_{n}(x)$ to $f(x)$. For this purpose we use the following result [4].

Proposition 5. If $s_{n}(x)$ is any cubic spline interpolating $f(x) \in C^{4}[a, b]$, then

$$
\max _{x_{i} \leqslant x \leqslant x_{i+1}}\left|s_{n}^{(j)}(x)-f^{(j)}(x)\right| \leqslant\left(1 / 2^{3-j}\right) h^{2-j} L_{i}, \quad j=0,1,2,
$$

where $L_{i}=(1 / 8) h^{2} k+\max \left\{\left|f^{\prime \prime}\left(x_{i}\right)-M_{i}\right|,\left|f^{\prime \prime}\left(x_{i+1}\right)-M_{i+1}\right|\right\}$ and $\left|f^{(4)}(x)\right| \leqslant k$, $x \in[a, b]$.

In view of Proposition 5 we have to determine $\alpha$ in such a way that the values $\left|f^{\prime \prime}\left(x_{i}\right)-M_{i}\right|$ are as small as possible.

Let $R$ be the $(n+1)$-vector whose components are $f^{\prime \prime}\left(x_{i}\right), i=0,1, \ldots, n$, and consider the vector $R-M$. Using the cubic spline condition (2.3) and the definition of $\tilde{A}$ in (2.9) yields

$$
\begin{aligned}
& R-M=\left(f^{\prime \prime}(a)-M_{0}, w^{T} \tilde{A}^{-1}, f^{\prime \prime}(b)-M_{n}\right)^{T}, \\
& w=\tilde{A} \tilde{R}-b+M_{0} e_{1}+M_{n} e_{n-1}, \\
& \tilde{R}=\left(f^{\prime \prime}\left(x_{1}\right), f^{\prime \prime}\left(x_{2}\right), \ldots, f^{\prime \prime}\left(x_{n-1}\right)\right)^{T} .
\end{aligned}
$$

Since, by Taylor's formula,

$$
\begin{array}{r}
f^{\prime \prime}\left(x_{i}\right)=\left(f\left(x_{i-1}\right)-2 f\left(x_{i}\right)+f\left(x_{i+1}\right)\right) / h^{2}+\left(h^{2} / 24\right) f^{(4)}\left(\xi_{i}\right)+\left(h^{2} / 24\right) f^{(4)}\left(\eta_{i}\right), \\
x_{i}<\xi_{i}<x_{i+1}, x_{i-1}<\eta_{i}<x_{i},
\end{array}
$$

we can write

$$
\begin{aligned}
\tilde{A} \tilde{R}-b= & \left(-2 f^{\prime \prime}\left(x_{1}\right)+f^{\prime \prime}\left(x_{2}\right)+\left(h^{2} / 2\right) \theta_{1},\right. \\
& f^{\prime \prime}\left(x_{1}\right)-2 f^{\prime \prime}\left(x_{2}\right)+f^{\prime \prime}\left(x_{3}\right)+\left(h^{2} / 2\right) \theta_{2}, \ldots, \\
& f^{\prime \prime}\left(x_{n-3}\right)-2 f^{\prime \prime}\left(x_{n-2}\right)+f^{\prime \prime}\left(x_{n-1}\right)+\left(h^{2} / 2\right) \theta_{n-2}, \\
& \left.f^{\prime \prime}\left(x_{n-2}\right)-2 f^{\prime \prime}\left(x_{n-1}\right)+\left(h^{2} / 2\right) \theta_{n-1}\right)^{T}, \\
\left|\theta_{i}\right| \leqslant k= & \max _{a \leqslant x \leqslant b}\left|f^{(4)}(x)\right| .
\end{aligned}
$$

Again using Taylor's formula, we find

$$
\begin{aligned}
f^{\prime \prime}\left(x_{i+1}\right)-2 f^{\prime \prime}\left(x_{i}\right)+ & f^{\prime \prime}\left(x_{i-1}\right)=\left(h^{2} / 2\right)\left(f^{(4)}\left(\tilde{\xi}_{i}\right)+f^{(4)}\left(\tilde{\eta}_{i}\right)\right), \\
& x_{i}<\tilde{\xi}_{i}<x_{i+1}, x_{i-1}<\tilde{\eta}_{i}<x_{i}, i=1,2, \ldots, n-1 .
\end{aligned}
$$


Therefore, we have

$$
\begin{aligned}
& \begin{array}{l}
w=\left(M_{0}-2 f^{\prime \prime}\left(x_{1}\right)+f^{\prime \prime}\left(x_{2}\right)+\left(h^{2} / 2\right) \theta_{1},(3 / 2) h^{2} \tilde{\theta}_{2}, \ldots,(3 / 2) h^{2} \tilde{\theta}_{n-2},\right. \\
\left.f^{\prime \prime}\left(x_{n-2}\right)-2 f^{\prime \prime}\left(x_{n-1}\right)+M_{n}+\left(h^{2} / 2\right) \theta_{n-1}\right)^{T}, \\
\left|\tilde{\theta}_{i}\right| \leqslant k .
\end{array}
\end{aligned}
$$

Now, from (3.5) and (3.6), the value of $\alpha$ for which we have the best uniform convergence on $[a, b]$ is $\alpha=-1$. In fact, in this case, since from Proposition 4

$$
\begin{aligned}
& M_{0}=f^{\prime \prime}(a)+(h / \sqrt{3}) f^{\prime \prime \prime}(a)+O\left(h^{2}\right), \\
& M_{n}=f^{\prime \prime}(b)+(h / \sqrt{3}) f^{\prime \prime \prime}(b)+O\left(h^{2}\right),
\end{aligned}
$$

we have

$$
\begin{aligned}
& \begin{array}{l}
R-M=\left(-(h / \sqrt{3}) f^{\prime \prime \prime}(a)+O\left(h^{2}\right), w^{T} \tilde{A}^{-1},-(h / \sqrt{3}) f^{\prime \prime \prime}(b)+O\left(h^{2}\right)\right)^{T} \\
w=\left((h / \sqrt{3}) f^{\prime \prime \prime}(a)+O\left(h^{2}\right),(3 / 2) h^{2} \tilde{\theta}_{2}, \ldots,\right. \\
\left.(3 / 2) h^{2} \tilde{\theta}_{n-2},(h / \sqrt{3}) f^{\prime \prime \prime}(b)+O\left(h^{2}\right)\right)^{T}
\end{array}
\end{aligned}
$$

Using relations (3.7), we can prove the following

Proposition 6. The spline function $s_{n}(x)$ obtained by (2.6) with $\alpha=-1$ fulfills the conditions:

$$
\left|s_{n}^{(i)}(x)-f^{(i)}(x)\right| \leqslant(h / 2)^{3-i}(\gamma+O(h)), \quad i=0,1,2, a \leqslant x \leqslant b,
$$

where $\gamma=\max \left(\left|f^{\prime \prime \prime}(a)\right|,\left|f^{\prime \prime \prime}(b)\right|\right)$.

Proof. Since

$$
\begin{aligned}
\left\|\tilde{A}^{-1}\right\| & =\left\|(1 / 4) \sum_{i=0}^{\infty}(-1)^{i+1} H^{i} / 4^{i}\right\| \\
& \leqslant(1 / 4) \sum_{i=0}^{\infty}\left(1 / 4^{i}\right)\|H\|^{i}=(1 / 4) \sum_{i=0}^{\infty} 1 / 2^{i}=1 / 2,
\end{aligned}
$$

we have $\left\|\tilde{A}^{-1} w\right\|<(1 / 2)\|w\|$. Therefore, from (3.7) we get

$$
\max _{i}\left|f^{\prime \prime}\left(x_{i}\right)-M_{i}\right| \leqslant(h / \sqrt{3}) \max \left(\left|f^{\prime \prime \prime}(a)\right|,\left|f^{\prime \prime \prime}(b)\right|\right)+O\left(h^{2}\right),
$$

which, in view of Proposition 5, completes the proof.

We are now looking for numbers $a^{\prime}, b^{\prime}$, such that $a<a^{\prime}<b^{\prime}<b$ and

$$
\max _{a^{\prime} \leqslant x \leqslant h^{\prime}}\left|s_{n}^{(i)}(x)-f^{(i)}(x)\right|=O\left(h^{4-i}\right) .
$$

For this purpose, observe that from (3.7) we have

$$
\begin{aligned}
& z=\tilde{A}^{-1} w=\left((h / \sqrt{3}) f^{\prime \prime \prime}(a)+O\left(h^{2}\right)\right) \tilde{A}^{-1} e_{1} \\
& +\left((h / \sqrt{3}) f^{\prime \prime \prime}(b)+O\left(h^{2}\right)\right) \tilde{A}^{-1} e_{n-1}+(3 / 2) h^{2} \tilde{A}^{-1} y \\
& \quad y=\left(0, \tilde{\theta}_{2}, \tilde{\theta}_{3}, \ldots, \tilde{\theta}_{n-1}, 0\right)^{T} .
\end{aligned}
$$


Moreover, from the proof of Lemma 3, we have that the elements of the first column of $\tilde{A}^{-1}$ are given by $(-1)^{i+1}\left(d^{i}-d^{2 n-i}\right) /\left(1-d^{2 n}\right), d=2-\sqrt{3}$. Therefore, since $\left\|\tilde{A}^{-1}\right\| \leqslant 1 / 2,\|y\| \leqslant k$ and $\tilde{A} \in \tau_{n-1}$, we have

$$
\left|z_{i}\right| \leqslant(3 / 4) h^{2} k+(h / \sqrt{3})(\gamma+O(h))\left(d^{i}-d^{2 n-i}+d^{n-i}-d^{n+i}\right) /\left(1-d^{2 n}\right),
$$

where $\gamma=\max \left\{\left|f^{\prime \prime \prime}(a)\right|,\left|f^{\prime \prime \prime}(b)\right|\right\}$.

Now, if $\log h / \log d \leqslant i \leqslant n-\log h / \log d$, then

$$
\left(d^{i}-d^{2 n-i}+d^{n-i}-d^{n+i}\right) /\left(1-d^{2 n}\right) \leqslant d^{i}+d^{n-i}+O\left(d^{n}\right) \leqslant 2 h+O\left(d^{n}\right) ;
$$

whence

$$
\left|z_{i}\right| \leqslant h^{2}((3 / 4) k+(2 / \sqrt{3}) \gamma)+O\left(h^{3}\right)
$$

if $\log h / \log d \leqslant i \leqslant n-\log h / \log d$.

Now we can apply Proposition 5 to get the following result.

Proposition 7. The spline function obtained by (2.6) with $\alpha=-1$ fulfills the conditions

$$
\begin{aligned}
\left|s_{n}^{(i)}(x)-f^{(i)}(x)\right| \leqslant\left(1 / 2^{3-i}\right) h^{4-i}((7 / 8) k+(2 / \sqrt{3}) \gamma+O(h)), & \\
& a^{\prime} \leqslant x \leqslant b^{\prime}, i=0,1,2 ; \\
\left|s_{n}^{(3)}(x)-f^{(3)}(x)\right| \leqslant 2 h(k+2 \gamma / \sqrt{3}), \quad a^{\prime} \leqslant x \leqslant b^{\prime}, & x \neq x_{j}, j=0,1, \ldots, n ;
\end{aligned}
$$

where $a^{\prime}-a=b-b^{\prime}=h \log h / \log d \quad$ and $k=\max _{a \leqslant x \leqslant b}\left|f^{(4)}(x)\right|, \quad \gamma=$ $\max \left\{\left|f^{\prime \prime \prime}(a)\right|,\left|f^{\prime \prime \prime}(b)\right|\right\}$.

Proof. In view of (3.9) and (3.5) we have

$$
\max _{a^{\prime} \leqslant x_{j} \leqslant h^{\prime}}\left|f^{\prime \prime}\left(x_{j}\right)-M_{j}\right| \leqslant h^{2}((3 / 4) k+(2 / \sqrt{3}) \gamma)+O\left(h^{3}\right) .
$$

Therefore, from Proposition 5, we have the result relative to $s_{n}^{(i)}(x)-f^{(i)}(x)$, $i=0,1,2$. In the case of the third derivative, observe that the function $g(x)=s_{n}^{\prime \prime}(x)$ $-f^{\prime \prime}(x)$ belongs to $C^{2}\left[x_{i}, x_{i+1}\right]$, therefore, applying Taylor's formula at the point $x \in\left[x_{i}, x_{i+1}\right]$ with increment

$$
\tilde{h}=\left\{\begin{aligned}
-h / 2 & \text { if } x>\left(x_{i+1}+x_{i}\right) / 2 \\
h / 2 & \text { if } x \leqslant\left(x_{i+1}+x_{i}\right) / 2
\end{aligned}\right.
$$

we get

$$
\begin{aligned}
s_{n}^{\prime \prime \prime}(x)-f^{\prime \prime \prime}(x)= & \left(s_{n}^{\prime \prime}(x+\tilde{h})-f^{\prime \prime}(x+\tilde{h})-s_{n}^{\prime \prime}(x)+f^{\prime \prime}(x)\right) / h \\
& -(\tilde{h} / 2) f^{(4)}(\xi),
\end{aligned}
$$

where $\xi$ belongs to the inverval with endpoints $x$ and $x+\tilde{h}$. Therefore,

$$
\left|s_{n}^{\prime \prime \prime}(x)-f^{\prime \prime \prime}(x)\right| \leqslant \max _{a^{\prime} \leqslant x \leqslant h^{\prime}}\left|s_{n}^{\prime \prime}(x)-f^{\prime \prime}(x)\right| / h+(h / 4) k \leqslant 2 h(k+(2 / \sqrt{3}) \gamma) \text {. }
$$

Final Remarks. We have shown that, among the splines interpolating to $f(x)$ and satisfying (2.6), the best one is the spline $S(x)$ minimizing the functional $F_{3}$. The computation of $S(x)$ can be performed according to the following steps:

(1) Compute $p_{1}=6 \sum_{i=1}^{n-1} v_{i} b_{i}, p_{2}=6 \sum_{i=1}^{n-1} v_{n-i} b_{i}$, (see (3.2));

(2) Compute $M_{0}$ and $M_{n}$ by means of (3.1);

(3) Solve the linear system $\tilde{A} M=b-M_{0} e_{1}-M_{n} e_{n-1}$. 
Compared to natural splines, here we have to compute $M_{0}$ and $M_{n}$ with a computational overhead of $2 n+O(1)$ multiplications. In this analysis we have assumed that the numbers $\varphi, \psi$ and $v_{i}$, which do not depend on the data $b_{i}$, are given constants which can be precomputed once and for all. Actually, in view of (2.11a), the evaluation of $\varphi$ and $\psi$ costs a linear time. Moreover, since the matrix vector product $F a$, where $F$ is the matrix in (2.8), can be computed by means of fast Fourier transform algorithms, the evaluation of $v=\tilde{A}^{-2} e_{1}=F D^{-2} F e_{1}, \quad D=$ $\operatorname{diag}(4+2 \cos (\pi i /(n+1)))$, can be carried out in $O(n \log n)$ arithmetic operations.

Dipartimento di Matematica

Università di Pisa

56100 Pisa, Italy

Dipartimento di Informatica

Università di Pisa

56100 Pisa, Italy

1. J. H. Ahlberg, E. N. Nilson \& J. L. Walsh, The Theory of Splines and Their Applications, Academic Press, New York, 1967.

2. D. Bini \& M. Capovani, "Spectral and computational properties of band symmetric Toeplitz matrices," Linear Algebra Appl., v. 52/53, 1983, pp. 99-126.

3. A. GHizzeTtI, "Interpolazione con splines verificanti una opportuna condizione," Calcolo, v. 20, 1983 , pp. 53-65.

4. D. KerShaw, "A note on the convergence of interpolatory cubic splines," SIAM J. Numer. Anal., v. 8, 1971, pp. 67-74.

5. J. StOER \& R. BUliRSCH, Introduction to Numerical Analysis, Springer, Berlin, 1980. 